\title{
Prediction of BRCA gene mutation status in epithelial ovarian cancer by radiomics models based on 2D and 3D CT images
}

Liu Mingzhu', Ge Yaqiong², Li Mengru³ and Wei Wei ${ }^{4^{*}}$

\begin{abstract}
Background: The objective of this study is to explore the value of two-dimensional (2D) and three-dimensional (3D) radiomics models based on enhanced computed tomography $(\mathrm{CT})$ images in predicting BRCA gene mutations in patients with epithelial ovarian cancer.

Methods: The clinical and imaging data of 106 patients with ovarian cancer confirmed by surgery and pathology were retrospectively analyzed and genetic testing was performed. Radiomics features extracted from the 2D and 3D regions of interest of the patients' primary tumor lesions were selected in the training set using the maximum correlation and minimum redundancy method. Then, the best features were selected through Lasso tenfold cross-validation. Feature subsets were employed to establish a radiomics model. The model's performance was evaluated via area under the receiver operating characteristic curve analysis and its clinical validity was assessed by using the model's decision curve.

Results: On the validation set, the area under the curve values of the 2D, 3D, and $2 \mathrm{D}+3 \mathrm{D}$ combined models were 0.78 (0.61-0.96), $0.75(0.55-0.92)$, and $0.82(0.61-0.96)$, respectively. However, the DeLong test $P$ values between the three pairs of models were all $>0.05$. The decision curve analysis showed that the radiomics model had a high net benefit across all high-risk threshold probabilities.
\end{abstract}

Conclusions: The three radiomics models can predict the BRCA gene mutation in ovarian cancer, and there were no statistically significant differences between the prediction performance of the three models.

Keywords: Ovarian cancer, BRCA gene, Radiomics, Mutation

\section{Introduction}

According to data from the International Cancer Research Center, approximately 52,000 new cases of ovarian cancer are diagnosed in China each year, with a high tumor mortality rate [1,2], and epithelial ovarian cancer accounts for $90 \%$ of all ovarian malignancies [3]. Approximately $50 \%$ of epithelial ovarian cancers exhibit

\footnotetext{
*Correspondence: weiweill@126.com

${ }^{4}$ Department of Radiology, Division of Life Sciences and Medicine, The First Affiliated Hospital of USTC, University of Science and Technology of China, Hefei 230001, Anhui, China

Full list of author information is available at the end of the article
}

DNA repair defects through homologous recombination, and BRCA1/2 mutations in germline and somatic cells are the most common mechanism underlying homologous recombination deficiency [4]. Patients with BRCA1/2 mutant ovarian cancer are sensitive to platinum drugs, have a higher progression-free survival period, and have better prognosis $[5,6]$. The main method for clinical judgment of BRCA gene mutations is genetic testing at present, but it is costly and time-consuming, and unit sampling cannot cover the entire tumor [7]. Nougaret et al. [8] predicted BRCA gene status by observing $\mathrm{CT}$ features and found that some radiomics 
features are related to gene mutation status, but the judgment of these imaging features was dependent on the observer's subjective experience.

Radiomics can objectively quantify the relationship between the pixels and the spatial distributions of medical images and fully explore the hidden information in the images that cannot be observed by the naked eyes. In previous studies, radiomics has been used to capture tumors' inherent heterogeneity and correlate it with potential gene expression types [9]. Extracting radiomics features requires delineating the region of interest (ROI) of the lesion, which is currently done by two commonly used methods: two-dimensional (2D) and threedimensional (3D) delineation. However, the advantages and disadvantages of these two delineation methods are still controversial $[10,11]$. Therefore, in this study, we explored the predictive value of radiomics models based on different delineation methods for BRCA gene mutations in patients with epithelial ovarian cancer, and compared the prediction performance of each model, to improve the prediction of gene mutation status. In doing so, we strived to seek a model that could provide an ideal and convenient method to sketch the area of interest.

\section{Methods}

Research subjects

We performed a retrospective analysis of patients with epithelial ovarian cancer who received treatment at our institution from March 2017 to July 2020. We collected clinical pathological data, CT enhanced images before surgery, radiotherapy, and chemotherapy, and postoperative pathological diagnosis. The inclusion criteria were as follows: (1) epithelial ovarian cancer pathologically confirmed by biopsy; (2) abdominal CT scan performed before the operation, and the images including the arterial, venous, and delayed phases; (3) no radiotherapy or chemotherapy performed before the operation; (4) lesion size $\geq 10 \mathrm{~mm}$. The exclusion criteria were as follows: (1) large image artifacts interfering with observation; (2) intolerance to enhanced CT examination; (3) preoperative radiotherapy and chemotherapy; (4) a history of other malignant tumors or pelvic metastases. In total, 106 patients were included in the study (Fig. 1). Among them, 63 cases, aged 35-75 years (average: 54.86 years), were in the BRCA gene non-mutation group; 43 cases aged 36-77 years (average: 53.93 years), were in the BRCA gene mutation group.

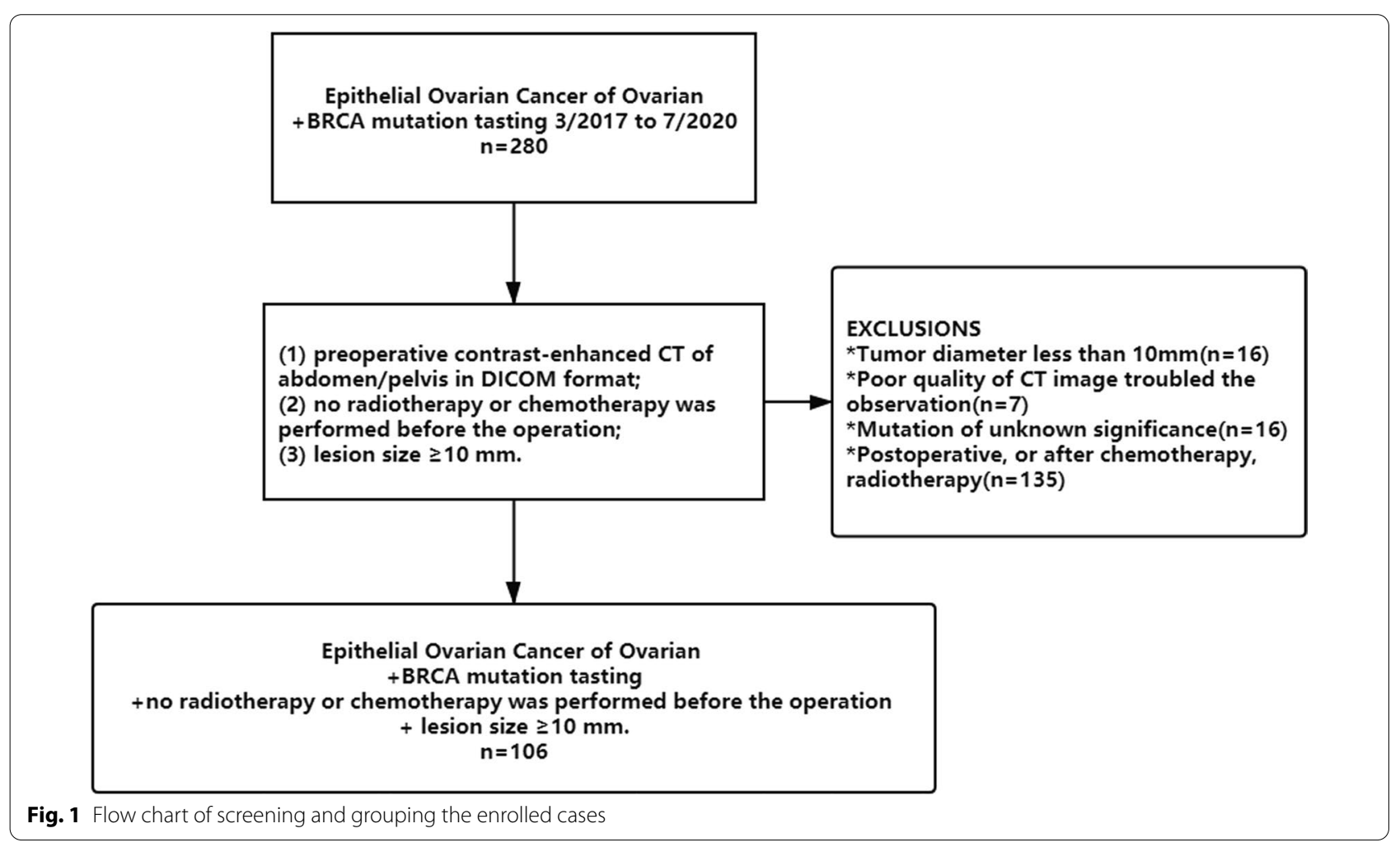




\section{Collection of general characteristics}

The clinical data of 106 patients, including patient's age, maximum tumor diameter and tumor markers, were collected and analyzed,.

\section{Genetic testing}

NGS genetic testing technology was applied on all patients, who had at least one of the following indications: (1) family history of pathogenic BRCA gene mutations; (2) family history of breast cancer before the age of 45 years or triple-negative breast cancer before the age of 60 years; (3) affordability of genetic testing or the doctor's requirement of genetic testing. To ensure the consistency of the test results, all subjects were tested by the same testing agency (Huada BGI).

\section{Image acquisition}

The patients fasted for more than $8 \mathrm{~h}$ and drank approximately $500-1000 \mathrm{~mL}$ of clean water orally $15-30 \mathrm{~min}$ before the examination. After the bladder was filled, the abdomen and pelvis were scanned using a GE Discovery CT 750HD (HDCT, USA) scanner in supine position. The scan ranged from the top of the diaphragm or the level of the iliac spine to the symphysis pubis. The tube voltage was $120 \mathrm{kV}$, the tube current was $280-300 \mathrm{~mA}$, the layer thickness was $5 \mathrm{~mm}$, the reconstruction dimension was $1.25 \mathrm{~mm}$, the layer spacing was $5 \mathrm{~mm}$, and the pitch was 1.375:1. The contrast agent for enhanced scanning was $1.5 \mathrm{~mL} / \mathrm{kg}$ of iohexol $(300 \mathrm{mgI} / \mathrm{mL})$, which was injected through the median cubital vein with a flow rate of $2.5-3.0 \mathrm{~mL} / \mathrm{s}$. The contrast agent was injected twice: at $25-30 \mathrm{~s}$ and $60-70 \mathrm{~s}$ for arterial and venous scanning, respectively.

\section{Image segmentation and feature extraction}

The preoperative abdominal enhanced CT image data of all patients were collected from the ICPACS workstation of the CT room at the Imaging Department of our institution and exported in.DICOM format. For all the image modality, the slices were resampled to $1^{*} 1^{*} 1 \mathrm{~cm}^{3}$, and the intensity range were normalized to a mean value of 0 and standard deviation of 1 (z score standardization). Doctor A, a senior diagnostic imaging doctor, used ITK-Snap software (https://www.itksnap.org) to delineate the (ROI) of the lesion in the third phase of enhanced CT. For 2D delineation, the scope included the layer with the largest lesion surface area, and for 3D delineation, the scope covered all possible areas of the target lesion. During the delineation, necrosis, blood vessels, and other structures were eliminated as completely as possible (Fig. 2). Two weeks later, doctor A randomly selected data of 30 patients to perform ROI delineation again to evaluate the intra-group consistency of ROI delineation (intra-ICC). To evaluate the between-group consistency of ROI delineation (inter-ICC), another radiologist, $\mathrm{B}$, also drew the ROIs on the 30 patients' images independently.

A.K. software (GE Healthcare, AnalysisKit; Version: 3.2.0.R) conforming to the IBSI standard was used to extract features from the ROIs outlined in each image. A total of 681 image biomarkers were obtained in each phase (non-mutation group labeled PA1-63, marked as 0; mutation group labeled PA64-106, marked as 1), including histogram, grayscale interconnected area matrix, grayscale co-occurrence matrix, morphology, run-length matrix, and wavelet change (wavelet) and Laplacian change (ln) characteristics.

\section{Feature screening and model establishment}

The features of the arterial phase, venous phase, and delay phase were integrated into 2043 features. The data were randomly divided into the training and test sets in a 7:3 ratio. In the training set, maximum correlation minimum redundancy (mRMR) and LASSO (least absolute shrinkage and selection operator) tenfold cross-validation, were used to select features. First, mRMR was performed to remove redundant and irrelevant features, incorporate the retained features into the LASSO regression, find the value of hyperparameter $\lambda$ that minimized the binomial deviation, and then retain the subset of features whose coefficients were not 0 to construct the final model.

\section{Statistical analysis and processing}

The chi-square test or Mann-Whitney U test was used to check for significant differences in general features and clinical characteristics between the two groups. The area under curve (AUC), model accuracy, precision, sensitivity, specificity, negative predictive value, and positive predictive value were analyzed to evaluate the radiomics models' predictive ability. Finally, decision curve analysis was used to evaluate the models' clinical applicability. All statistical analyses were performed using R software (version 3.6.1, https://www.r-project.org).

For general clinical characteristics, we used the Chisquare test and the Kruskal-Wallis $\mathrm{H}$ test to analyze abnormally distributed continuous variables. $\mathrm{P}$ values $<0.05$ were considered statistically significant. All statistical analyses were performed using R software (version 3.6.1) and the Python programming language (version 3.5.6).

The intraclass correlation coefficients (ICCs) of each texture feature were calculated to evaluate the withinand between-observer changes in the texture features extracted by the ROI segmentation and to explain each feature's reproducibility, according to the following scale: 


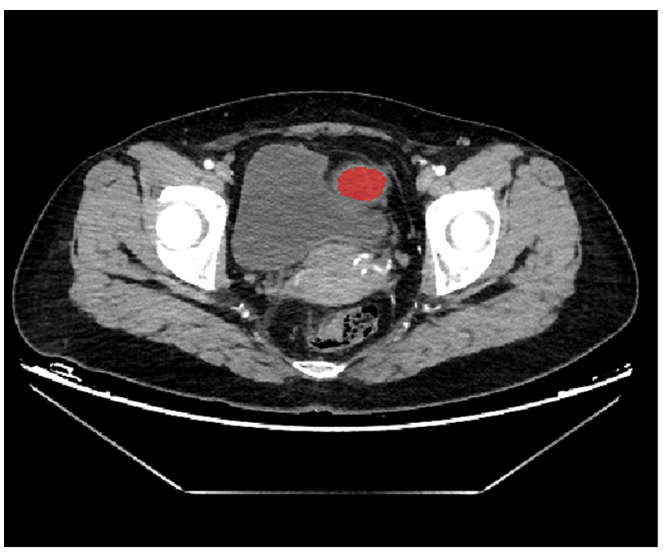

(a)

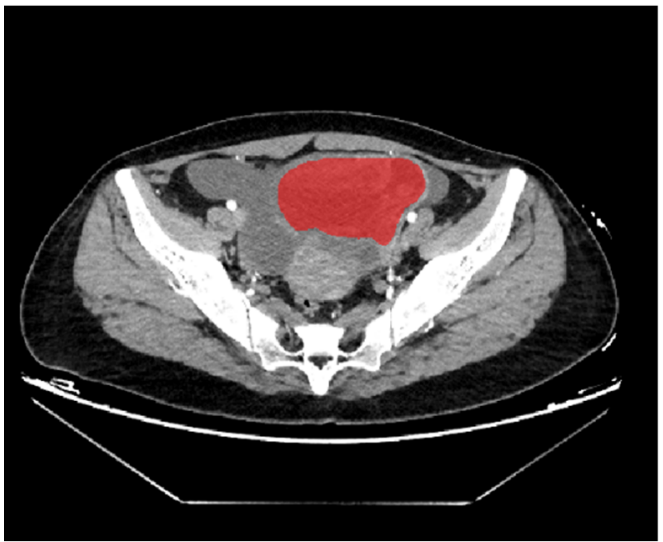

(c)

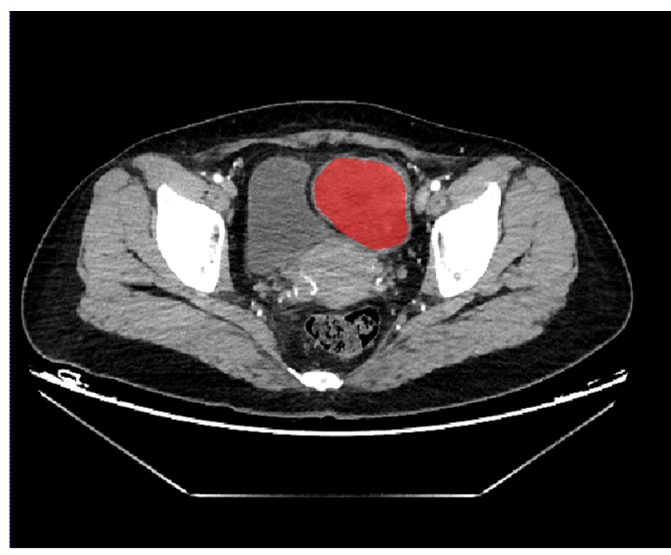

(b)

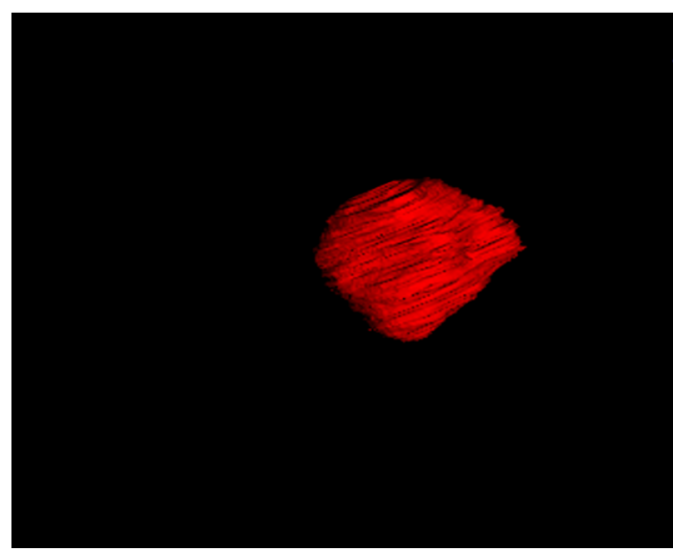

(d)

Fig. 2 a--d Images of a 35-year-old patient with high-grade (stage IIIC) serous carcinoma of the ovary, which was genetically detected with a non-BRCA gene mutation. ITK-SNAP software was used to segment and label the lesion layer-by-layer to generate a 3D image of the lesion

$\mathrm{ICC}<0.4$, poor; $0.59>\mathrm{ICC} \geq 0.4$, fair; $0.75>\mathrm{ICC} \geq 0.6$, good; and ICC $\geq 0.75$, excellent. Features with ICC $\geq 0.8$ were considered stable and included in further analyses.

\section{Results}

\section{General characteristics}

No statistically significant differences in age composition, maximum tumor diameter, or tumor markers were found among the three groups $(P>0.05$; Table 1$)$.

\section{Construction and verification of prediction models Feature screening}

The ICC ranges within and between the 2D-outlined feature data groups were $(-0.18-1.00)$ and $(-0.08-1.00)$, respectively. In total, 531 features had ICC values of $>0.75$ across the two groups. In the 3D-outlined feature data group, the ICC ranges within and between groups were $(-0.06-1.00)$ and $(-0.40-1.00)$, respectively. In total, 548 features had ICC values of $>0.75$ across the two groups.

Table 1 General characteristics of the three groups

\begin{tabular}{|c|c|c|c|c|c|c|}
\hline Variable & Sample & Class meaning unknown & Class mutation & Class wild & Statistics & $P$ value \\
\hline Age & 106 & $55.00(49.00,58.05)$ & $52.00(47.70,57.20)$ & $54.00(48.00,59.30)$ & 0.781 & 0.677 \\
\hline Maximum_Diameter & 106 & $8.75(5.95,11.00)$ & $7.00(4.57,9.24)$ & $7.00(4.49,10.00)$ & 2.929 & 0.231 \\
\hline Tumor marker CA125 & 86 & $15(83.33 \%)$ & $42(93.33 \%)$ & $63(81.82 \%)$ & 3.601 & 0.463 \\
\hline Tumor marker CA125 、 CA199 & 19 & $3(16.67 \%)$ & $3(6.67 \%)$ & $13(16.88 \%)$ & & \\
\hline Tumor marker CA199 & 1 & $0(0.00 \%)$ & $0(0.00 \%)$ & $1(1.30 \%)$ & & \\
\hline
\end{tabular}


We then used the mRMR and LASSO regression models to perform feature screening on features with ICC values of $>0.75$ in the $2 \mathrm{D}, 3 \mathrm{D}$, and $2 \mathrm{D}+3 \mathrm{D}$ models. For the $2 \mathrm{D}$ images, the binomial deviation was smallest at the optimal tuning parameter value of $\ln \lambda=0.0118$, and the four features whose coefficients were not 0 at that value were retained. For the 3D images, the binomial deviation was smallest at the optimal tuning parameter value of $\ln$ $\lambda=0.0279$, and the 13 features whose coefficients were not 0 at that value were retained. For the $2 \mathrm{D}+3 \mathrm{D}$ images, the binomial deviation was the smallest at the optimal tuning parameter value of $\ln \lambda=0.0294$ (Fig. 3a), and the 12 features whose coefficients were not 0 at that value (Fig. 3b) were retained; eight and four were retained from 2 and 3D images, respectively. The corresponding coefficients of the features are shown in Fig. 3c.

\section{Diagnostic efficiency of the devised models}

Logistic regression was used to establish radiomics models using the selected features, and the radscore of each case was calculated. Table 2 shows the radscore distribution in the gene mutation and wild-type groups generated by the $2 \mathrm{D}, 3 \mathrm{D}$, and $2 \mathrm{D}+3 \mathrm{D}$ models. We used the AUC of the receiver operating characteristic curve and the models' accuracy, sensitivity, specificity, negative predictive value, and positive predictive value to evaluate their predictive ability.

In the training set, the $2 \mathrm{D}$ model's image AUC was 0.81 (0.71-0.91), the 3D model's image AUC was 0.80 (0.700.90 ), and the $2 \mathrm{D}+3 \mathrm{D}$ model's image AUC was 0.91 (0.84$0.97)$. In the test set, the $2 \mathrm{D}$ model's image AUC was 0.78 (0.61-0.96), the 3D model's image AUC was 0.75 (0.550.92 ), and the $2 \mathrm{D}+3 \mathrm{D}$ model's image AUC was 0.82 (0.670.98) (Table 3). The highest AUC value of the $2 \mathrm{D}+3 \mathrm{D}$ model indicated its highest diagnostic efficacy (Fig. 4). However, the DeLong test results showed that the P values among the three pairs of models were all $>0.05$, suggesting that there were no statistically significant differences in prediction efficiency among the three pairs of models.

Finally, a decision curve was used to evaluate the three models' clinical effectiveness (Fig. 5). As the radiomics model's standard net benefit within a range of the highrisk threshold increased, its clinical effectiveness increased. The decision curves show that the clinically effective performance of the 2D+3D model was much higher than those of the other two models.

The above results indicate that the radiomics model based on 2D and 3D images can effectively predict BRCA gene mutations in patients with epithelial ovarian cancer, although the 2D+3D model showed no significantly different predictive performance from that of the radiomic models based on 2D or 3D images alone.

\section{Conclusions}

Ovarian cancer has the highest mortality rate among gynecological malignancies. More than $70 \%$ of patients with ovarian cancer are already in the advanced stage when detected [12], and there is still a lack of accurate early diagnosis and prevention methods [13]. The use of radiomics can avoid the disadvantages of conventional imaging examinations such as subjectivity, histopathology, and local sampling.

Guo Jianlin et al. [14] found that the combined radiomics model of arterial phase and venous phase had higher predictive performance than the separate model of each phase. Using the CT features of 539 cases of lung adenocarcinoma, Yooh et al. [15] analyzed the tumor size, location, volume, density, CT value, and the relationship between pixel-based texture features and gene expression patterns and found that these image features showed good discrimination of fusion-positive from fusion-negative lung adenocarcinomas. In this study, the quantitative features were selected and the prediction model was established using radiomics features extracted from the arterial phase, venous phase, and delay phase of enhanced $\mathrm{CT}$ images, and the resulting radiomics scores were strongly correlated with the BRCA gene mutation $(r=0.65, \mathrm{P}<0.001$; Fig. 3c). Totally, 2,043 radiomics features were extracted from the $\mathrm{CT}$ images of 106 patients with epithelial ovarian cancer, Twelve characteristics were finally selected and the model was constructed via multivariate logistic regression. Among them, the $\mathrm{P}$ values of the 3D_Artery_wavelet.HLL firstorder_Kurtosis, 3D-Delay-wavelet.LHH_IDMN, Venous_wavelet.LLL_firstorder_Range, Delay_wavelet.HHH_glszm_SmallAreaHighGrayLevelEmphasis, Venous_wavelet.HHH_firstorder_MeanAbsoluteDeviation, and 2D_Artery_wavelet.HHH_glszm_ZoneEntropy

(See figure on next page.)

Fig. 3 Application of LASSO (Least absolute shrinkage and selection operator)-logistic regression to imaging feature screening in the 2D + 3D model shows that the LASSO-logistic regression model selects tuning parameters $(\lambda)$ through tenfold cross-validation and obtains the relationship between binomial variance and logarithm $(\lambda)(\mathbf{a})$. The relationship is retained with the parameters that yield the smallest binomial deviation, and the 12 best features with non-zero coefficients $(\mathbf{b})$ are retained in the final model. The relationships between the features and gene mutation status (correlation coefficient $\times 100$ ) are shown in the heat map (c) 


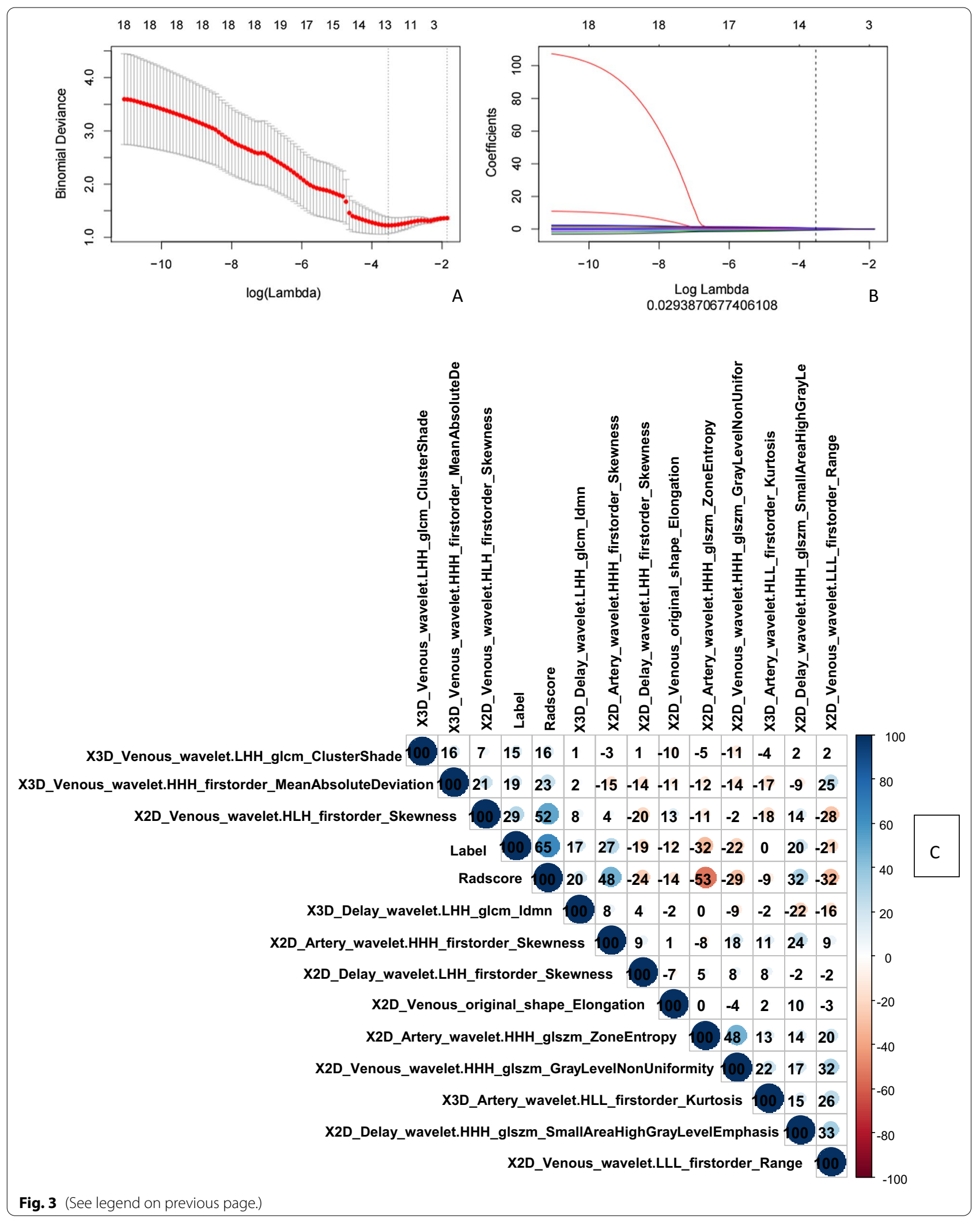


Table 2 Radscores of each patient in the training and validation sets calculated by different models, and their distribution and difference statistics in patients with wild-type and mutant type BRCA genes

\begin{tabular}{lllllrr}
\hline & Variable & Sample & Wild & Mutation & Statistics & $\boldsymbol{P}$ value \\
\hline Train & Radscore_2D3D & 76 & $-1.22 \pm 1.06$ & $0.52 \pm 0.94$ & -7.329 & $<0.001$ \\
& Radscore_3D & 76 & $-0.71(-1.06,-0.28)$ & $0.00(-0.36,0.28)$ & -4.413 & $<0.001$ \\
& Radscore_2D & 76 & $-0.51 \pm 0.23$ & $-0.19 \pm 0.30$ & -5.303 & $<0.001$ \\
\multirow{2}{*}{ Test } & Radscore_2D3D & 30 & $-1.03(-2.32,-0.01)$ & $0.66(-0.23,4.92)$ & -2.963 & 0.003 \\
& Radscore_3D & 30 & $-0.61(-0.95,-0.25)$ & $-0.04(-0.48,0.49)$ & -2.159 & 0.031 \\
& Radscore_2D & 30 & $-0.41 \pm 0.22$ & $-0.17 \pm 0.23$ & -2.9 & 0.007 \\
\hline
\end{tabular}

Table 3 Cutoff value prediction ability of the 2D, 3D, and 2D +3D joint radiomics models

\begin{tabular}{llllllll}
\hline & AUC & Accuracy & Sensitivity & Specificity & Positive value & Negative value & Cut-off \\
\hline 2D Training set & $0.81(0.71-0.91)$ & $0.75(0.63-0.84)$ & 0.68 & 0.83 & 0.86 & 0.65 & -0.43 \\
2D validation set & $0.78(0.61-0.96)$ & $0.73(0.54-0.87)$ & 0.61 & 0.91 & 0.91 & 0.61 & -0.65 \\
3D training set & $0.80(0.70-0.90)$ & $0.75(0.63-0.84)$ & 0.71 & 0.81 & 0.84 & 0.70 & 0.92 \\
3D validation set & $0.75(0.55-0.92)$ & $0.74(0.55-0.88)$ & 0.84 & 0.58 & 0.76 & 0.61 \\
2D + 3D Training set & $0.91(0.84-0.97)$ & $0.86(0.77-0.93)$ & 0.95 & 0.74 & 0.84 & 0.14 \\
2D + 3D validation set & $0.82(0.67-0.98)$ & $0.73(0.54-0.87)$ & 0.611 & 0.91 & 0.91 & \\
\hline
\end{tabular}

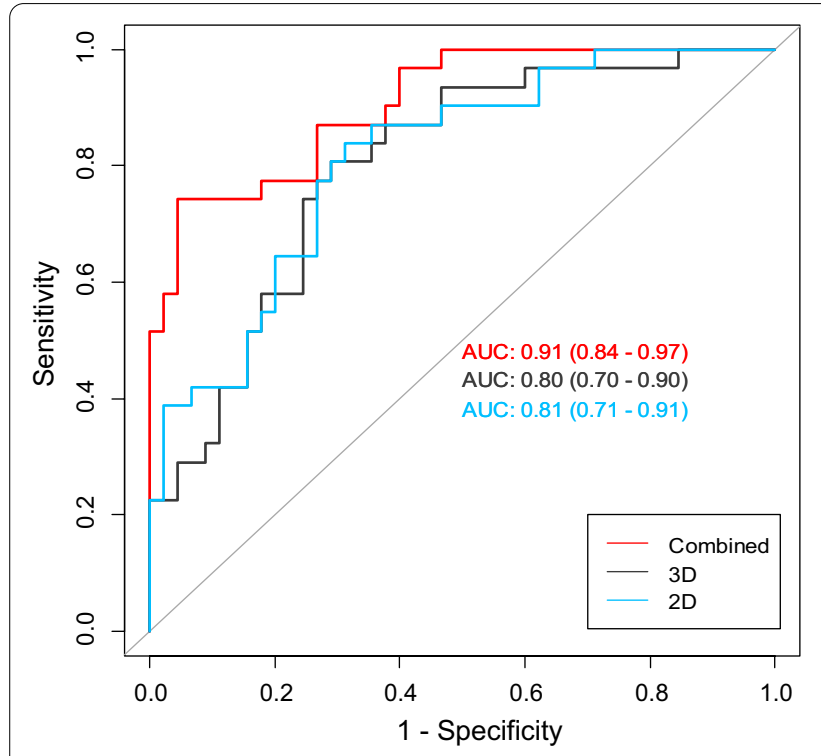

A

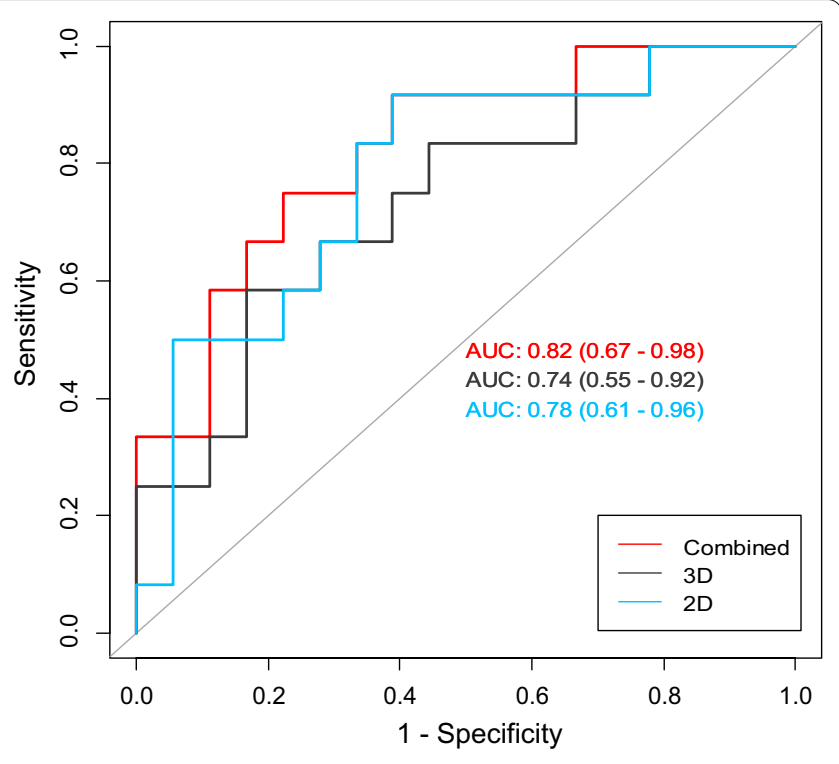

B

Fig. 4 ROC curves of 2D model, 3D model, and 2D +3D model in the training group (a) and validation group (b)

were less than 0.05 , and can be used as independent risk predictors. The values of these characteristics in the mutation group were higher than those in the nonmutation group. For example, Artery_wavelet.HLL_firstorder_Kurtosis is a measure of the "peak" of the value distribution in the image ROI transferred by wavelet from the artery phase. Higher kurtosis means that the quality of the distribution is concentrated in the tail instead of the average, which can be used as a label to predict the mutation status of the BRCA gene. Other detailed explanation of the features are in the appendix (Table 4). The radscore obtained from the mutlvirate logistic 


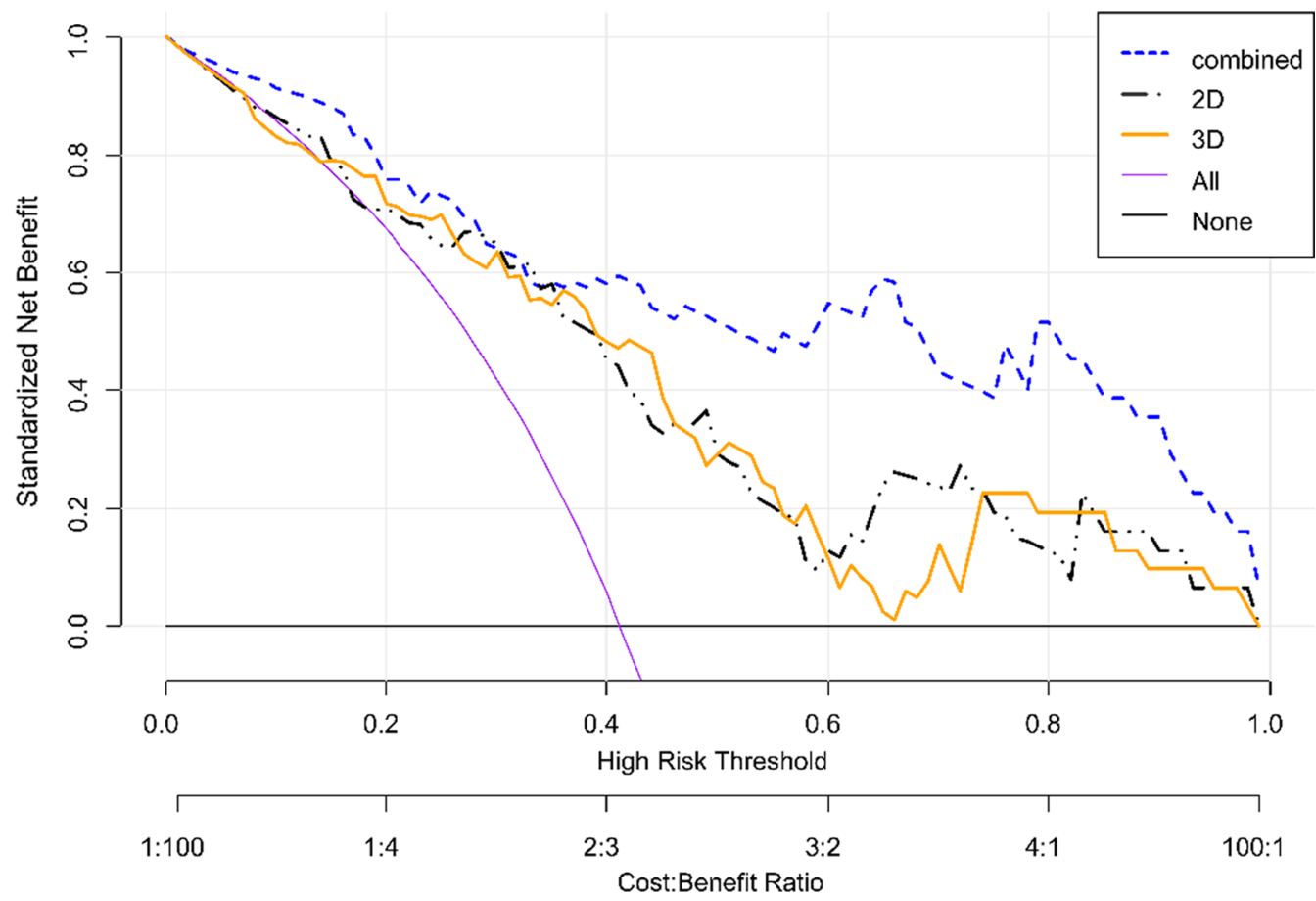

Fig. 5 The yellow line, black dotted line, and blue dotted line represent the data obtained from the 2D, 3D, and 2D+3D images, respectively. The $x$-axis represents the patient's personal threshold probability (e.g., $x=0.6$ means that the high-risk threshold of ovarian cancer and BRCA gene mutation is 60\%). The $y$-axis represents net income. The line labeled "All" represents the hypothesis that all ovarian cancer cases are caused by BRCA gene mutations. The thin line labeled "None" represents the assumption that there are no BRCA gene mutations in patients with ovarian cancer

regression model was also higher in the mutation group than the wild group in both the $2 \mathrm{D}$ and $3 \mathrm{D}$ and $2 \mathrm{D}+3 \mathrm{D}$ $\operatorname{model}(\mathrm{p}<0.05)$. Therefore, the radiomics signature established by the selected radiomics feature radscore can be used as a biomarker for BRCA gene mutation prediction.

The study by Lei $\mathrm{Xu}$ et al. found that in both univariate and multivariate analyses, $3 \mathrm{D}$ image features showed better prediction performance than 2D image features [16]. However, according to Shen et al. [17], 2D features had better performance. Yet, Lifeng Yang et al. [18] found that the features derived from a 2D+3D model showed better prognostic performance than $2 \mathrm{D}$ or $3 \mathrm{D}$ features alone in predicting overall survival of non-small cell lung cancer. Therefore, we established prediction models using 2D, 3D, and $2 \mathrm{D}+3 \mathrm{D}$ image features, and found that the AUC values of all three models were over 0.75 , and all three models' exhibited good prediction performance, indicating that the radiomics models can effectively predict BRCA gene mutation status in patients with epithelial ovarian cancer. The $2 \mathrm{D}+3 \mathrm{D}$ model had the highest AUC value, but the DeLong test results showed no statistical differences in predictive performance between the three pairs of models. The results showed that $2 \mathrm{D}$ outlining of the layer with the largest lesion diameter could cover the central region of the tumor and achieve high predictive performance in predicting BRCA gene mutations in ovarian cancer. The 3D outlining images could provide information about tumors' heterogeneity outside the central area, but this information could not contribute to the prediction of BRCA gene mutations in the present study. Therefore, we believe that when the image is delineated in 2D, with appropriate selection of lesion delineation level, the predictive performance the radiomics model is close to that of the $2 \mathrm{D}+3 \mathrm{D}$ model. This insight may improve the practical efficiency of establishing radiomics model in clinical practice.

This study had some limitations. First, this study was a single-center retrospective study. The included cases were all from our hospital and therefore lacked external verification. Second, the sample size was small, which may lead to bias in the data. Third, all ROIs in this study were drawn manually, making the results easily affected by subjective factors. Lastly, in this study, there were 63 and 43 patients in the wild-type and mutant groups, respectively. The data of the two groups were unbalanced, and the model may therefore be biased. 
Table 4 The detailed information of the features

\begin{tabular}{ll}
\hline Image type & Features \\
\hline X3D-venous-wavelet.LHH & Glcm-cluster shade \\
& $\sum_{i=1}^{N_{g}} \sum_{j=1}^{N_{g}}\left(i+j-\mu_{X}-\mu_{y}\right)^{3} p(i, j)$ \\
& Firstorder-mean absolute deviation (MAD) \\
X3D-venous-wavelet.HHH & $\frac{1}{N_{p}} \sum_{i=1}^{N_{p}}|X(i)-X|$ \\
X2D-venous-wavelet.HLH X2D-delay- & Firstorder-skewness \\
wavelet.LHHX2D-Artery-wavelet.HHH & $\frac{\mu_{3}}{\sigma^{3}}=\frac{\frac{1}{N_{p}} \sum_{i=1}^{N_{p}}(X(i)-X)^{3}}{\left(\sqrt{\frac{1}{N_{p}}} \sqrt{\sum_{i=1}^{N_{p}}(X(i)-X)^{2}}\right)^{3}}$ \\
& \\
& \\
& \\
X3D-delay-wavelet.LHH & $\sum_{k=0}^{N_{g}-1} \frac{P_{X-y}(k)}{1+\left(\frac{k^{2}}{N_{g}^{2}}\right)}$
\end{tabular}

X2D-venous

X2D-venous-wavelet.HHH

X2D-venous-wavelet.HHH

X3D-artery-wavelet.HLL

X2D-delay-wavelet.HHH

GIszm-small area high gray level emphasis (SAHGLE) $\frac{\sum_{j=1}^{N g} \sum_{j=1}^{N_{s}} \frac{P(i, j) i^{2}}{j^{2}}}{N_{z}}$

\section{Features explanation}

Cluster Shade is a measure of the skewness and uniformity of the GLCM. A higher cluster shade implies greater asymmetry about the mean

Mean Absolute Deviation is the mean distance of all intensity values from the Mean Value of the image array

Skewness measures the asymmetry of the distribution of values about the Mean value. Depending on where the tail is elongated and the mass of the distribution is concentrated, this value can be positive or negative

IDMN (inverse difference moment normalized) is a measure of the local homogeneity of an image. IDMN weights are the inverse of the Contrast weights (decreasing exponentially from the diagonal $\mathrm{i}=\mathrm{ji}=\mathrm{j}$ in the GLCM). Unlike Homogeneity2, IDMN normalizes the square of the difference between neighboring intensity values by dividing over the square of the total number of discrete intensity values

Elongation shows the relationship between the two largest principal components in the ROI shape. For computational reasons, this feature is defined as the inverse of true elongation

ZE measures the uncertainty/randomness in the distribution of zone sizes and gray levels. A higher value indicates more heterogeneneity in the texture patterns

GLN measures the variability of gray-level intensity values in the image, with a lower value indicating more homogeneity in intensity values

Kurtosis is a measure of the 'peakedness' of the distribution of values in the image ROI. A higher kurtosis implies that the mass of the distribution is concentrated towards the tail(s) rather than towards the mean. A lower kurtosis implies the reverse: that the mass of the distribution is concentrated towards a spike near the Mean value

SAHGLE measures the proportion in the image of the joint distribution of smaller size zones with higher graylevel values

The range of gray values in the $\mathrm{RO}$ 
In summary, the $2 \mathrm{D}, 3 \mathrm{D}$, and $2 \mathrm{D}+3 \mathrm{D}$ radiomics models based on enhanced CT images can effectively predict BRCA gene mutation status in patients with epithelial ovarian cancer and provide a new type of method for clinical evaluation of patients' genetic mutation status.

\section{Acknowledgements}

We thank Richard Lipkin, PhD, from Liwen Bianji, Edanz Group China (www. liwenbianji.cn/ac), for editing the English text of this manuscript.

\section{Authors' contributions}

LM: Conception and design, collection and assembly of data, validation, and writing of original draft. GY: Data analysis and interpretation. LM: Methodology. WW: Administrative support, provision of study materials or patients, and supervision. All authors read and approved the final manuscript.

\section{Funding}

This work is supported by the National Natural Science Youth Science Fund Project (No. 81501468), by the Fundamental Research Funds for the Central Universities (No. WK9110000002). Application and clinical promotion of pathological classification and FIGO staging of epithelial ovarian cancer based on CT imaging-genomics, supported by Chinese Red Cross Foundation: Leading Elite Scientific Research Project, (XM_HR_YXFN_2021_05_19, 2., June 2021 to June 2022).

\section{Availability of data and materials}

The datasets used and/or analyzed in the current study are available from the corresponding author on reasonable request.

\section{Declarations}

\section{Ethics approval and consent to participate}

Our institutional review board (IRB) waived written informed consent for this retrospective study which evaluated de-identified data and involved no potential risk to patients. The method used in this study was in accordance with the medical Research Ethics Committee of the First Affiliated Hospital of University of Science and Technology of China (No. 2021-RE-078).

\section{Consent for publication}

All authors agree to publish.

\section{Competing interests}

All authors have completed the ICMJE uniform disclosure form. The authors have no conflicts of interest to declare. We declare that we do not have any commercial or associative interest that represents a conflict of interest in connection with the work submitted.

\section{Author details}

${ }^{1}$ Division of Life Sciences and Medicine, The First Affiliated Hospital of University of Science and Technology of China, University of Science and Technology of China, Hefei 230031, Anhui, China. ${ }^{2}$ GE Healthcare China, Pudong New Area, No.1 Huatuo Road, Shanghai 210000, China. ${ }^{3}$ Department of Radiology, The First Affiliated Hospital of University of Science and Technology of China, Hefei 230031, Anhui, China. ${ }^{4}$ Department of Radiology, Division of Life Sciences and Medicine, The First Affiliated Hospital of USTC, University of Science and Technology of China, Hefei 230001, Anhui, China.

Received: 14 July 2021 Accepted: 17 November 2021

Published online: 26 November 2021

\section{References}

1. Siegel RL, Miller KD, Jemal A. Cancer statistics, 2020. CA Cancer J Clin. 2020;70(1):7-30.

2. Bray F, Ferlay J, Soerjomataram I, Rebecca L, Lindsey A, Jemal A. Global cancer statistics 2018: CL OBOCAN estimates of incidence and mortality worldwide for 36 cancers in 185 countries. CA Cancer J Clin. 2018;68(6):394-424.

3. Kim J, Coffey DM, Creighton CJ, Yu Z, Hawkins SM, Matzuk MM. High grade serous ovarian cancer arises from fallopian tube in a mouse model. Proc Natl Acad Sci U S A. 2012;109(10):3921-6.

4. Konstantinopoulos PA, Ceccaldi R, Shapiro GI, D'Andrea AD. Homologous recombination deficiency: exploiting the fundamental vulnerability of ovarian cancer. Cancer Discov. 2015;5(11):1137-54.

5. Byrski T, Huzarski T, Dent R, Marczyk E, Jasiowka M, Gronwald J, Jakubowicz J, Cybulski C, Wisniowski R, Godlewski D, Lubinski J, Narod SA. Pathologic complete response to neoadjuvant cisplatin in BRCA1-positive breast cancer patients. Breast Cancer Res Treat. 2014;147:401-5.

6. Tutt A, Tovey H, Cheang MCU, Kernaghan S, Kilburn L, Gazinska P, et al. Carboplatin in BRCA1/2-mutated and triple-negative breast cancer BRCAness subgroups: the TNT Trial. Nat Med. 2018;24:628-37.

7. Vargas HA, Huang EP, Lakhman Y, Ippolito JE, Bhosale P, Mellnick V, Shinagare AB, Anello M, Kirby J, Fevrier-Sullivan B, Freymann J, Jaffe CC, Sala E. Radiogenomics of high-grade serous ovarian cancer: multireader multiinstitutional study from the cancer genome atlas ovarian cancer imaging research group. Radiology. 2017;285(2):482-92.

8. Nougaret S, Lakhman Y, Gönen M. High-grade serous ovarian cancer: associations between BRCA mutation status, CT imaging phenotypes, and clinical outcomes. Radiology. 2017;285(2):472-81.

9. Aerts HJ, Velazquez ER, Leijenaar RT, Parmar C, Grossmann P, Carvalho S, Bussink J, Monshouwer R, Haibe-Kains B, Rietveld D, Hoebers F, Rietbergen MM, Leemans CR, Dekker A, Quackenbush J, Gillies RJ, Lambin P. Decoding tumour phenotype by noninvasive imaging using a quantitative radiomics approach. Nat Commun. 2014;5:4006.

10. Zhao $\mathrm{H}$. et al. TCGA-TCIA-based CT radiomics study for noninvasively predicting epstein-barr virus status in gastric cancer. AJR, 2021, 11.

11. Lei $X$, et al. A multi-organ cancer study of the classification performance using 2D and 3D image features in radiomics analysis. Phys Med Biol. 2019;64(21):215009.

12. Chien J, Poole EM. Ovarian cancer prevention, screening, and early detection: report from the 11 th biennial ovarian cancer research symposium. Int J Gynecol Cancer, 2017, 27(9S Suppl 5): S20-S22.

13. Modugno F. Ovarian cancer and high-risk women symposium presenters Ovarian cancer and high-risk women-implications for prevention, screening, and early detection. Gynecol Oncol. 2003;91(1):15-31.

14. Chen X, Yang Z, Yang J, Liao Y, Pang P, Fan W, Chen X. Radiomics analysis of contrast-enhanced CT predicts lymphovascular invasion and disease outcome in gastric cancer: a preliminary study. Cancer Imaging. 2020;20(1):24.

15. Hyun JY, et al. Decoding tumor phenotypes for ALK, ROS1, and RET fusions in lung adenocarcinoma using a radiomics approach. Medicine. 2015;94(41):e1753.

16. Xu L, Yang P, Yen EA, Wan Y, Jiang Y, Cao Z, Shen X, Wu Y, Wang J, Luo C, Niu T. A multi-organ cancer study of the classification performance using 2D and 3D image features in radiomics analysis. Phys Med Biol. 2019;64(21):215009.

17. Shen C, Liu Z, Guan M, Song J, Lian Y, Wang S, Tang Z, Dong D, Kong $L$, Wang M, Shi D, Tian J. 2D and 3D CT radiomics features prognostic performance comparison in non-small cell lung cancer. Transl Oncol. 2017;10(6):886-94.

18. Yang L, Yang J, Zhou X, Huang L, Zhao W, Wang T, Zhuang J, Tian J. Development of a radiomics nomogram based on the 2D and 3D CT features to predict the survival of non-small cell lung cancer patients. Eur Radiol. 2019;29(5):2196-206.

\section{Publisher's Note}

Springer Nature remains neutral with regard to jurisdictional claims in published maps and institutional affiliations. 